\title{
A Yang-Mills-Higgs Monopole of Charge 2
}

\author{
R. S. Ward \\ Department of Mathematics, Trinity College, Dublin, Ireland
}

\begin{abstract}
A new static, purely magnetic Yang-Mills-Higgs monopole solution is presented. It is axisymmetric and has a topological charge of 2; the charge is located at a single point.
\end{abstract}

\section{Introduction}

This paper is concerned with Yang-Mills-Higgs monopoles which are static and purely magnetic, in the Prasad-Sommerfield limit [1]. This means that we have a Higgs field $\phi$ and a gauge potential $A_{j}(j=1,2,3)$ on Euclidean 3-space $\mathbb{R}^{3}$, satisfying the following five requirements.

(i) $\phi \& A_{j}$ take values in the Lie algebra of SU(2). In other words, $\phi$ and $A_{j}$ have the form $\phi=\phi^{a} \sigma^{a}, A_{j}=A_{j}^{a} \sigma^{a}$, where $\sigma^{a}$ are the Pauli matrices, and where $\phi^{a}, A_{j}^{a}$ are scalar functions on $\mathbb{R}^{3}$ which in some gauge are real-valued. (We shall be allowing $\mathrm{SL}(2, \mathbb{C})$-valued gauge transformations, so $\sigma^{a} \& A_{j}^{a}$ will not be realvalued in every gauge.)

(ii) In some gauge, $\phi \& A_{j}$ are smooth (say $C^{\infty}$ ) on $\mathbb{R}^{3}$.

(iii) The Bogomolny equations

$$
G_{j k}^{a}=-\varepsilon_{j k \ell} D_{\ell} \phi^{a}
$$

are satisfied, where

$$
\begin{gathered}
G_{j k}^{a}=\partial_{j} A_{k}^{a}-\partial_{k} A_{j}^{a}+\kappa \varepsilon^{a b c} A_{j}^{b} A_{k}^{c}, \\
D_{j} \phi^{a}=\partial_{j} \phi^{a}+\kappa \varepsilon^{a b c} A_{j}^{b} \phi^{c},
\end{gathered}
$$

$\kappa$ being some real number (the coupling constant).

(iv) The norm $\|\phi\|=\left(\phi^{a} \phi^{a}\right)^{1 / 2}$ of the Higgs field has the asymptotic behaviour

$$
\|\phi\|=1-m / r+0\left(r^{-2}\right) \text { as } r \rightarrow \infty,
$$

where $r$ is the Euclidean distance from the origin in $\mathbb{R}^{3}$, and $m$ is some real number.

(v) The energy

$$
E=\int\left(\frac{1}{4}\|G\|^{2}+\frac{1}{2}\|D \phi\|^{2}\right) d^{3} x
$$

is finite. Here $\|G\|^{2}=G_{j k}^{a} G_{j k}^{a}$ and $\|D \phi\|^{2}=\left(D_{j} \phi^{a}\right)\left(D_{j} \phi^{a}\right)$. 


\section{Remarks}

(a) $\|\phi\|,\|G\|$ and $\|D \phi\|$ are invariant under $\operatorname{SL}(2, \mathbb{C})$ gauge transformations.

(b) Requirements (i)-(iv) in fact imply requirement (v). To see this, first note that the Bogomolny equation enables one to rewrite $E$ as

$$
E=\int\|D \phi\|^{2} d^{3} x .
$$

From the Bogomolny equation and the Bianchi identities $D_{[j} G_{k \ell]}=0$ (square brackets denote skew-symmetrization) we get $D_{j} D_{j} \phi=0$, and from this it follows that $\|D \phi\|^{2}=\frac{1}{2} \partial_{j} \partial_{j}\|\phi\|^{2}$. Now letting $B_{R}$ denote the three-dimensional closed ball with radius $R$ in $\mathbb{R}^{3}$, we can write

$$
\begin{aligned}
E & =\lim _{R \rightarrow \infty} \int_{B_{R}} \frac{1}{2} \partial_{j} \partial_{j}\|\phi\|^{2} d^{3} x \\
& =\lim _{R \rightarrow \infty} \int_{\partial B_{R}} \frac{1}{2} \partial_{j}\|\phi\|^{2} d^{2} S^{j} \text { (Stokes' theorem) } \\
& =4 \pi m
\end{aligned}
$$

from requirement (iv). (The author is indebted to L. O'Raifeartaigh for supplying this argument.)

(c) The topological charge $n$ is defined by [2]

$$
n=\lim _{R \rightarrow \infty} \frac{1}{8 \pi} \int_{\partial B_{R}} \xi_{j} d^{2} S^{j},
$$

where $\xi_{j}=\varepsilon_{j k \ell} \varepsilon_{a b c} \hat{\phi}^{a} \partial_{k} \hat{\phi}^{b} \partial_{\ell} \hat{\phi}^{c}$ and $\hat{\phi}^{a}=\|\phi\|^{-1} \phi^{a}$. The number $n$ is necessarily an integer [2]. Its value is unchanged if we replace $\xi_{j}$ by $-\kappa \varepsilon_{j k \ell} F_{k \ell}$, where $F_{k \ell}$ is the 't Hooft magnetic tensor (see [2] Eq. (1), but beware the sign error contained therein). Now using the standard expression for $F_{j k}([2]$, Eq. (1a)), the bound $\|D \phi\|=O\left(r^{-2}\right)$ which follows from finiteness of energy, the Bogomolny equations, and the asymptotic form of $\|\phi\|$, one easily deduces that

$$
n=m \kappa \text {. }
$$

The magnetic charge is defined to be $n / \kappa$, and is therefore equal to $m$.

$\mathrm{Up}$ to now only one monopole solution was known: the spherically-symmetric Bogomolny-Prasad-Sommerfield (BPS) monopole, which has $n=1$ [1]. One line of attack on the problem of finding further solutions was provided by the realization that the Bogomolny equations are equivalent to the self-dual YangMills equations in Euclidean 4-space with the added condition that everything be independent of imaginary time; [3]. Indeed, the equations

$$
\frac{1}{2} \varepsilon_{\mu \nu \alpha \beta} G_{\alpha \beta}^{a}=G_{\mu \nu}^{a}, \partial_{0} A_{\mu}^{a}=0
$$

are equivalent to

$$
G_{j k}^{a}=-\varepsilon_{j k \ell} D_{\ell} A_{0}^{a}, \quad \partial_{0} A_{\mu}^{a}=0 ;
$$

and these are exactly the Bogomolny equations in 3-space, if we interpret $A_{0}$ as the Higgs field $\phi$. Manton [3] recognized that the BPS solution can be obtained out of the well-known "'t Hooft ansatz", which expresses $A_{\mu}$ as functional of a 
scalar superpotential satisfying a linear equation. But he discovered that the ansatz does not produce any other monopole solutions.

Now the 't Hooft ansatz is the first in a sequence of analogous ansätze $\mathscr{A}_{1}$, $\mathscr{A}_{2}, \ldots$ [4], which arose out of the "twistor" approach to the self-dual Yang-Mills equations [5]. Corrigan et al. [6] pointed out the existence of Bäcklund transformations relating these ansätze to one another, and Lohe [7] tried to construct a monopole with $n=3$ by applying two successive Bäcklund transformations to the BPS solution. But this approach was unsuccessful $[7,8]$.

A more recent approach $[9,10]$ was that of using the AHDM instanton construction [11], which also arose out of twistor theory. Again, the BPS solution can be obtained via this procedure, but no new solutions have so far been produced. It should be remarked that a recent existence theorem due to Taubes [12] has shown that there are many multimonopole solutions waiting to be found.

The approach employed in this paper is to use the ansätze $\mathscr{A}_{\ell}$ referred to above. In particular, we shall see that $\mathscr{A}_{2}$ produces a new solution which has $n=2$ and is axisymmetric. It is algebraically rather complicated and is presented here in a complex gauge ([3]: $\mathscr{A}_{1}$ produces the BPS monopole in a complex gauge). The point is that we can be certain, by arranging things appropriately, that there exists a gauge in which $\phi^{a}$ and $A_{j}^{a}$ are real-valued, even if we do not know explicitly how to transform into that gauge. Of course, all gauge-invariant objects like $\|\phi\|,\|D \phi\|, F_{j k}$ etc. will be real, if and when we compute them.

It is worth emphasizing the following point. The ansätze $\mathscr{A}_{\ell}$ are often described in terms of the Bäcklund transformations referred to above. This approach leads to difficulties with reality conditions and singularities. However, if one does not use the approach of Bäcklund transformations, then these difficulties are avoided. This paper illustrates that point.

\section{The Construction}

We shall follow the notation and use the results of Corrigan et al. [6]. The essential point of the twistor construction is that self-dual Yang-Mills fields correspond to certain holomorphic (i.e. complex-analytic) vector bundles over twistor space $[5,6$, Sect. 2]. Such a vector bundle is specified by a transition matrix $g(\omega, \pi)$ ([6], Eq. (2.10)). Here $g$ is a 2-by-2 matrix of functions of the four complex variables $\left(\omega_{1}, \omega_{2}, \pi_{1}, \pi_{2}\right) ; g$ is required to be holomorphic away from $\pi_{1}=0$ and $\pi_{2}=0$, and homogeneous of degree zero. A gauge potential $A_{\mu}$ can be extracted from $g\left([5,6]\right.$, Eqs. (2.11)-(2.14)), and this $A_{\mu}$ automatically satisfies the self-dual YangMills equations. Furthermore, every self-dual Yang-Mills field can be obtained in this way. In general, there is no known procedure for explicitly obtaining $A_{\mu}$ from $g$ (the "extraction" referred to above is somewhat implicit). However, if $g$ is equivalent to a matrix of the form

$$
\tilde{g}=\left[\begin{array}{cc}
\zeta^{\ell} & \rho(\omega, \pi) \\
0 & \zeta^{-\ell}
\end{array}\right],
$$

where $\zeta=\pi_{1} / \pi_{2}$ and $\ell$ is some positive integer, then we have an explicit formula 
for $A_{\mu}$ in terms of an integral of the function $\rho$. This formula is exactly the ansatz $\mathscr{A}_{\ell}[4,6]$. The word "equivalent" used above means the following: there exist matrices $\Lambda$ and $\Xi$, both taking values in $\mathrm{SL}(2, \mathbb{C})$, with $\Lambda$ holomorphic away from $\pi_{1}=0$ and $\Xi$ holomorphic away from $\pi_{2}=0$, such that

$$
\tilde{g}=\Lambda g \Xi \text {. }
$$

Our problem here is that we want to find a Yang-Mills-Higgs field satisfying the five requirements listed in Sect. 1. Condition (iii) is easily taken care of: since the self-dual Yang-Mills equations are automatically satisfied in our construction, we only need the additional condition $\partial_{0} A_{\mu}=0$, and this is achieved by requiring that $g$ depend on $\omega_{1}$ and $\omega_{2}$ only through the combination

$$
\gamma=-i \omega_{1} / \pi_{1}+i \omega_{2} / \pi_{2} \text {. }
$$

(It is easy, from the details given in [6], to see that this is sufficient.)

Thus, using the fact that $g$ is homogeneous, we may write $g=g(\gamma, \zeta)$, where $\zeta=\pi_{1} / \pi_{2}$. The requirement $(\mathrm{i})$ is achieved by imposing the conditions

$$
\begin{gathered}
\operatorname{det}(g)=1, \\
{[g(\gamma, \zeta)]^{*}=g\left(\bar{\gamma},-\bar{\zeta}^{-1}\right),}
\end{gathered}
$$

where* denotes conjugate transpose. That these conditions are sufficient is proved in the appendix. It is worth pointing out that one should think of $\gamma$ as ranging over the complex line $\mathbb{C}$, and $\zeta$ as ranging over the extended complex line, i.e. the Riemann sphere; recall that $-\bar{\zeta}^{-1}$ and $\zeta$ are antipodal points on the Riemann sphere.

Requirement (ii) (smoothness) essentially amounts to the condition that the function $\rho$ appearing in the matrix $\tilde{g}$ should be "nowhere-vanishing", in a certain sense which will be explained later. The boundary conditions (iv) and (v) amount to some condition on $g$, but it is unclear exactly what this condition is, so at present we just have to proceed by trial and error.

Our task, therefore, is to search for matrices $g$ which satisfy the above requirements, and which are equivalent to matrices $\tilde{g}$ of the form (1). The obvious startingpoint is to find the matrix $g_{\mathrm{BPS}}$ which generates the BPS solution. What one gets is the following. Let $\tilde{g}_{\mathrm{BPS}}$ be defined by

$$
\tilde{g}_{\mathrm{BPS}}=\left[\begin{array}{cc}
\zeta & \gamma^{-1}\left(e^{2 \mu}-e^{2 v}\right) \\
0 & \zeta^{-1}
\end{array}\right],
$$

where $\mu=i \omega_{2} / \pi_{2}$ and $v=i \omega_{1} / \pi_{1}$ (so $\gamma=\mu-v$ ). Now multiply $\tilde{g}_{\mathrm{BPS}}$ on the left and on the right by

$$
\left[\begin{array}{cc}
e^{-v} & 0 \\
0 & e^{v}
\end{array}\right] \text { and }\left[\begin{array}{cc}
0 & -e^{\mu} \\
e^{-\mu} & \zeta \gamma e^{-\mu}
\end{array}\right]
$$

respectively (notice that the left-hand matrix is holomorphic away from $\pi_{1}=0$, and the right-hand one way from $\pi_{2}=0$, as required). This gives

$$
g_{\mathrm{BPS}}=\left[\begin{array}{cc}
\gamma^{-1}\left(e^{\gamma}-e^{-\gamma}\right) & -\zeta e^{-\gamma} \\
\zeta^{-1} e^{-\gamma} & \gamma e^{-\gamma}
\end{array}\right],
$$

and this matrix depends only on $\gamma \& \zeta$ and satisfies (3), (4). One may now apply 
the results of $([6]$, Sect. 3) to verify that this choice of matrix does indeed correspond to the BPS solution (albeit in an unusual gauge: [3]). The value of the coupling constant used in [6] is $\kappa=2$, so for example one finds that

$$
\|\phi\|=\operatorname{coth}(2 r)-\frac{1}{2 r},
$$

as expected (i.e. $m=\frac{1}{2}, \kappa=2, n=1, E=2 \pi$ ).

Now how is one to find new solutions? Manton [3] showed that matrices of the form (1) with $\ell=1$ give nothing new, so clearly the thing to do is to take $\ell=2$, and to search for a function $\rho$ such that

$$
\tilde{g}=\left[\begin{array}{cc}
\zeta^{2} & \rho \\
0 & \zeta^{-2}
\end{array}\right]
$$

is equivalent to a matrix $g$ satisfying our requirements. One choice of $\rho$ that might work is $\rho=H^{-1}\left(e^{2 \mu}+e^{2 v}\right)$, where $H=\gamma^{2}+4 c^{2}, c$ being some real, nonzero constant. For then multiplying $\tilde{g}$ on the left and on the right by

$$
\left[\begin{array}{cc}
e^{-v} & 0 \\
0 & e^{\nu}
\end{array}\right] \text { and }\left[\begin{array}{cc}
0 & -e^{\mu} \\
e^{-\mu} & \zeta^{2} H e^{-\mu}
\end{array}\right]
$$

respectively, yields

$$
g(\gamma, \zeta)=\left[\begin{array}{cc}
H^{-1}\left(e^{\gamma}+e^{-\gamma}\right) & \zeta^{2} e^{-\gamma} \\
\zeta^{-2} e^{-\gamma} & H e^{-\gamma}
\end{array}\right]
$$

which satisfies the reality condition (4).

If we take this function $\rho$ and compute the Yang-Mills-Higgs field that it gives rise to, using the formulae in [6], we obtain the following.

Define a function $\Lambda$ on $\mathbb{R}^{3}$ by

$$
-2 \pi \Lambda=\cosh (2 R)+\cosh (2 \bar{R}),
$$

where $R^{2}=r^{2}-c^{2}+2 i c z, r^{2}=x^{2}+y^{2}+z^{2}$ and $(x, y, z)$ are the standard coordinates on $\mathbb{R}^{3}$. Note that there is no ambiguity as a result of taking the squareroot of $R^{2}$, since cosh is an even function. From now on let $c$ denote the constant $\pi / 4$. The functions $\Delta_{-1}, \Delta_{0} \& \Delta_{1}$ of $[6]$ are given by

$$
\begin{aligned}
\Delta_{-1} & =\bar{\xi}^{-1}\left(2 \Lambda-\Lambda_{z}\right) e^{2 i t}, \\
\Delta_{0} & =2 \bar{\xi}^{-1} \Lambda_{\xi} e^{2 i t}, \\
\Delta_{1} & =\xi^{-1}\left(2 \Lambda+\Lambda_{z}\right) e^{2 i t},
\end{aligned}
$$

where $\xi=x+i y, t=x^{0}$ and the subscripts denote partial differentiation. (Note that the $\Delta_{r}$ depend on $x^{0}$ : this dependence will disappear at the last step, when one computes $A_{j} \& \phi ;[3]$.)

Next define three scalar functions $E, F$ and $G$ by

$$
\begin{aligned}
& E=(\operatorname{det})^{-1} e^{2 i t} \Delta_{1}, \\
& F=-(\operatorname{det})^{-1} e^{2 i t} \Delta_{0}, \\
& G=(\operatorname{det})^{-1} e^{2 i t} \Delta_{-1},
\end{aligned}
$$


where det $=\Delta_{1} \Delta_{-1}-\left(\Delta_{0}\right)^{2}$. Finally, $\phi, A_{z}, A_{x}=A_{\xi}+A_{\bar{\xi}}$ and $A_{y}=i A_{\xi}-i A_{\bar{\xi}}$ are given by

$$
\begin{aligned}
\phi & =\frac{1}{2 F}\left[\begin{array}{cc}
F_{z} & -2 E_{\xi} \\
-2 G_{\bar{\xi}} & -F_{z}
\end{array}\right] \\
A_{z} & =\frac{i}{F}\left[\begin{array}{cc}
F & -E_{\xi} \\
G_{\bar{\xi}} & -F
\end{array}\right] \\
A_{\xi} & =\frac{-i}{2 F}\left[\begin{array}{cc}
F_{\xi} & 0 \\
G_{z}+2 G & -F_{\xi}
\end{array}\right] \\
A_{\bar{\xi}} & =\frac{i}{2 F}\left[\begin{array}{cc}
F_{\bar{\xi}} & E_{z}-2 E \\
0 & -F_{\bar{\xi}}
\end{array}\right] .
\end{aligned}
$$

This, then, is our candidate for a new solution, and we must now make sure that it satisfies the five requirements listed in Sect. 1. Conditions (i) and (iii) automatically hold, by construction. General theory says that condition (ii) will be satisfied provided that $\Delta_{0}, \Delta_{1} \& \Delta_{-1}$ are smooth and that det is nowhere-vanishing (this was what was meant by the earlier statement that $\rho$ should be "nowherevanishing"). In our case, we do not even need this theorem, since one can check directly (without too much trouble) that $\phi \& A_{j}$ as defined above are smooth (in fact real-analytic). As for condition (iv), we shall see in the next section that $\|\phi\|=1-1 / r+O\left(r^{-3}\right)$ as $r \rightarrow \infty$, so (iv) is satisfied with $m=1$. Condition (v) of course follows from the others.

Our construction has therefore yielded a monopole solution with topological charge $n=m \kappa=2$.

\section{Discussion}

When one tries to write out the fields $\phi^{a}$ and $A_{j}^{a}$ in terms of the coordinates $x, y, z$, one discovers that the expressions for them are rather complicated (and complexvalued). We know that there is a gauge in which the fields are real-valued, but at present this gauge is not known explicitly. It might be that there exists a gauge in which the fields are relatively simple functions, but this remains to be seen. Let us content ourselves here with a few remarks about the structure of the Higgs field $\phi$.

The matrix $\phi$ has the form

$$
\phi=\left[\begin{array}{cc}
U & V e^{-2 i \psi} \\
W e^{2 i \psi} & -U
\end{array}\right],
$$

where $U, V \& W$ are real-valued functions of $r$ and $z$, and where $\xi=|\xi| e^{i \psi}$. Thus $\phi$ is axisymmetric (about the $z$-axis) [13]. The appearance of the integer 2 in (7) supports the earlier assertion that the topological charge is 2 [2].

By way of illustration, here are the functions $U, V \& W$ on the $z$-axis and on the $z=0$ plane. On the axis,

$$
\begin{aligned}
& U=z\left(z^{2}+c^{2}\right)^{-1}-\tanh (2 z), \\
& V=W=0
\end{aligned}
$$


while on the plane,

$$
\begin{gathered}
U=0, \\
V=W=\frac{c^{2} \cosh (2 a)[\sinh (2 a)-2 a \cosh (2 a)]}{a\left[a^{2}-c^{2} \sinh ^{2}(2 a)\right]}-1,
\end{gathered}
$$

where $a=\left(r^{2}-c^{2}\right)^{1 / 2}$.

In the previous section we needed an asymptotic expression for $\|\phi\|$, up to and including terms of order $r^{-1}$. It is not difficult to obtain this by direct calculation. The author has used the computing system REDUCE 2 to calculate $\|\phi\|$ up to terms which fall off exponentially as $r \rightarrow \infty$. The result is

$$
\|\phi\|=1-a /\left(a^{2}+b^{2}\right)+0\left(e^{-4 r}\right)
$$

where $a$ and $b$ are defined by $(a+i b)^{2}=R^{2}=r^{2}-c^{2}+2 i c z, a>0$. So certainly $\|\phi\|=1-r^{-1}+0\left(r^{-3}\right)$, as claimed earlier.

By definition, the monopole is located at the point(s) where $\phi$ vanishes. We see from (8), (9) that $\phi$ vanishes at the origin $r=0$, and has no other zeros on the $z$-axis or $z=0$ plane. So if $\phi$ has any other zeros, they must (because of the symmetry of $\phi$ ) consist of rings surrounding the $z$-axis. The author has checked, by numerical computation, that this does not happen: our monopole is therefore located at a single point.

In the previous section, the constant $c$ was assumed to have the specific value $\pi / 4$. It turns out that no other value of $c$ gives a non-singular monopole; there are gauge-invariant singularities whenever $c \neq \pi / 4$. So our monopole solution depends on exactly five parameters, corresponding to the position of the monopole and the direction of its axis of symmetry.

It appears very likely that the techniques described in this paper can be used to obtain yet more monopole solutions, of arbitrarily high charge. This possibility is now being investigated.

\section{Appendix}

It has to be shown that if the matrix $g$ satisfies the conditions

$$
\begin{gathered}
\operatorname{det}(g)=1, \\
{[g(\gamma, \zeta)]^{*}=g\left(\bar{\gamma},-\bar{\zeta}^{-1}\right),}
\end{gathered}
$$

then the gauge potential $A_{\mu}\left(x^{j}\right)$ obtained from $g$ takes values in the Lie algebra of SU(2). The procedure by which $A_{\mu}$ is obtained is as follows. First, substitute $\gamma=\gamma\left(x^{j}, \zeta\right)=\xi \zeta-2 z-\bar{\xi} \zeta^{-1}$ into $g$ and "split" $g$ into two matrices $h$ and $k$ :

$$
g(\gamma(x, \zeta), \zeta)=h(x, \zeta) k(x, \zeta)^{-1}
$$

where $h$ is analytic away from $\zeta=0$ and $k$ away from $\zeta=\infty$. Then define $A_{\mu}(x)$ by

$$
\begin{aligned}
A_{P 1}-\zeta A_{P 2} & =-i h(x, \zeta)^{-1} D_{P}(\zeta) h(x, \zeta) \\
& =-i k(x, \zeta)^{-1} D_{P}(\zeta) k(x, \zeta)
\end{aligned}
$$


where $D_{P}(\zeta)$ is the differential operator

$$
D_{P}(\zeta)=\frac{\partial}{\partial x^{P 1}}-\zeta \frac{\partial}{\partial x^{P 2}}
$$

and the four coordinates $x^{P Q}(P, Q=1,2)$ are defined by

$$
\left[\begin{array}{ll}
x^{11} & x^{12} \\
x^{21} & x^{22}
\end{array}\right]=\left[\begin{array}{cc}
x^{0}-i z & -i \xi \\
-i \xi & x^{0}+i z
\end{array}\right] .
$$

See [6] for more details.

If $\operatorname{det}(g)=1$, then $h$ and $k$ may be chosen to have unit determinant as well, and it follows from (13) that trace $\left(A_{\mu}\right)=0$. It remains to be proved that in some gauge, $A_{\mu}$ is Hermitian: $A_{\mu}^{*}=A_{\mu}$.

Observe that $\gamma\left(x,-\bar{\zeta}^{-1}\right)=\overline{\gamma(x, \zeta)}$, so that Eqs. (11) and (12) imply

$$
k(x, \zeta)^{*-1} h(x, \zeta)^{*}=h\left(x,-\bar{\zeta}^{-1}\right) k\left(x,-\bar{\zeta}^{-1}\right)^{-1}
$$

and so

$$
h(x, \zeta)^{*} k\left(x,-\bar{\zeta}^{-1}\right)=k(x, \zeta)^{*} h\left(x,-\bar{\zeta}^{-1}\right)
$$

Now the left-hand side of (14) is antiholomorphic away from $\zeta=0$, and the righthand side away from $\zeta=\infty$. Thus by Liouville's theorem, both sides are constant in $\zeta$, i.e. both sides of (14) are equal to a matrix-valued function $\lambda(x)$.

The splitting (12) does not determine $h$ and $k$ uniquely: there is the freedom

$$
\begin{gathered}
h(x, \zeta) \mapsto h(x, \zeta) \Lambda(x), \\
k(x, \zeta) \mapsto k(x, \zeta) \Lambda(x),
\end{gathered}
$$

where $\Lambda(x)$ is an $\operatorname{SL}(2, \mathbb{C})$ matrix. This freedom corresponds exactly to the gauge freedom in the gauge field. Under the transformation (15) the matrix $\lambda(x)$ transforms as

$$
\lambda \mapsto \Lambda^{*} \lambda \Lambda \text {. }
$$

From the fact that $\lambda(x)$ equals both sides of (14), with $\zeta$ an arbitrary parameter, one easily sees that $\lambda=\lambda^{*}$. It follows that we can choose $\Lambda$ such that after the transformation (16) we have

$$
\lambda= \pm I
$$

$I$ being the identity 2-by-2 matrix. From now on we shall suppose that such a gauge transformation has been made.

In terms of the coordinates $x^{P Q}$, the Hermiticity condition $A_{\mu}^{*}=A_{\mu}$ is

$$
A_{11}^{*}=A_{22}, A_{21}^{*}=-A_{12},
$$

which is equivalent to

$$
\left[A_{11}-\zeta A_{12}\right]^{*}=\bar{\zeta}\left[A_{21}-\left(-\bar{\zeta}^{-1}\right) A_{22}\right] .
$$

This in turn in equivalent to

$$
\left[k(\zeta)^{-1} D_{1}(\zeta) k(\zeta)\right]^{*}=-\bar{\zeta}\left[h\left(-\bar{\zeta}^{-1}\right)^{-1} D_{2}\left(-\bar{\zeta}^{-1}\right) h\left(-\bar{\zeta}^{-1}\right)\right]
$$


because of (13). Now expand the left-hand side of (18), making use of the identities

$$
\begin{aligned}
& \overline{D_{1}(\zeta)}=\bar{\zeta} D_{2}\left(-\bar{\zeta}^{-1}\right), \\
& k(\zeta)^{*}= \pm h\left(-\bar{\zeta}^{-1}\right)^{-1} ;
\end{aligned}
$$

one then obtains the right-hand side of (18), as required.

Acknowledgement. The author is indebted to L. O'Raifeartaigh for valuable discussions.

\section{References}

1. Prasad, M. K., Sommerfield, C. M. : Phys. Rev. Lett. 35, 760-762 (1975)

2. Arafune, J., Freund, P. G. O., Goebel, C. J.: J. Math. Phys. (NY) 16, $433-437$ (1975)

3. Manton, N. S. : Nucl. Phys. B135, 319-332 (1978)

4. Atiyah, M. F., Ward, R. S. : Commun. Math. Phys. 55, 117-124 (1977)

5. Ward, R. S. : Phys. Lett. A61, 81-82 (1977)

6. Corrgian, E. F., Fairlie, D. B., Yates, R. G., Goddard, P. : Commun. Math. Phys. 58, 223-240 (1978)

7. Lohe, M. A. : Nucl. Phys. B142, 236-252 (1978)

8. Bruce, D. J. : Nucl. Phys. B142, 253-257 (1978)

9. Nahm, W. : Phys. Lett. B90, 413-414 (1980)

10. Nahm, W. : Phys. Lett. B93, 42-46 (1980)

11. Atiyah, M. F., Hitchin, N. J., Drinfeld, V. G., Manin, Yu. I. : Phys. Lett. A65, 185-187 (1978)

12. Jaffe, A., Taubes, C. H. : Vortices and monopoles. Boston: Birkhäuser 1980

13. Houston, P., O’Raifeartaigh, L. : Phys. Lett. B93, 151-154 (1980)

Communicated by A. Jaffe

Received September 8, 1980

\section{Notes added in proof.}

1. P. Houston and L. O'Raifeartaigh have proved that for any axisymmetric monopole system, the Higgs field $\phi$ vanishes at precisely one point. In particular, there can be no "rings" of charge. [Preprint DIAS-STP-80-39, Dublin.].

2. M. K. Prasad has explicitly found the gauge transformation which makes the solution real. He has also presented candidate solutions of arbitrary topological charge. [MIT Preprint, to appear in Commun. Math. Phys.].

3. P. Forgács, Z. Horváth and L. Palla have independently constructed the solution described in this paper, using what appears to be a different method. [Preprint KFKI-1980-122, Budapest.]. 
